\title{
POLÍTICAS DE EDUCACIÓN SUPERIOR Y PRODUCCIÓN DE CONOCIMIENTOS EN MÉxico (1980-2011)*
}

\author{
Armando Alcántara Santuario \\ Universidad Nacional Autónoma de México (UNAM). \\ Yuri Jiménez Nájera \\ Universidad Pedagógica Nacional (UPN).
}

RESUMEN: En este artículo se revisan diversos procesos e indicadores relacionados con la educación superior (ES) y la producción de conocimientos en México durante las últimas tres décadas. Los datos analizados indican que, a pesar de la rápida expansión de la ES mexicana durante el período 1980-2011, la productividad en investigación y la producción de conocimientos es muy baja, no sólo en comparación con las naciones más avanzadas, sino con países de la región. El crecimiento acelerado de la ES es un fenómeno mundial, aunque es más visible en algunas regiones que en otras. Comparativamente, México y Brasil muestran un fuerte incremento en términos absolutos, pero todavía sus niveles de cobertura son de tipo medio. Los indicadores que relacionan la capacidad científica, tecnológica y de innovación, en el caso de México, muestran una baja inversión con relación al PIB. Los estudiantes de posgrado han aumentado significativamente en los últimos años, pero siguen siendo pocos con respecto al número total. La cantidad de doctores graduados anualmente en México representa una cuarta parte de los graduados en Brasil. La comunidad de investigadores es muy reducida, tanto a nivel mundial como regional. Pese al aumento de artículos publicados en revistas internacionales, su número es aún pequeño. El coeficiente de inventiva presenta, además, una tendencia a la baja. Aun cuando los indicadores son insuficientes y limitados, hay lugar para el optimismo moderado debido al crecimiento en el número de estudiantes en ES y posgrado, así como

\footnotetext{
* Artigo recebido em 31/05/2012 e aprovado em 20/08/2012.
} 
las perspectivas de mayor inversión gubernamental en ciencia, tecnología e innovación.

Palabras-Claves: Educación Superior. Investigación. Producción de conocimiento.

...el conocimiento, producto principal de la ciencia, constituye un bien público indispensable para la sociedad y el acceso al mismo debe considerarse un derecho humano fundamental.

(Hacia una agenda nacional de ciencia, tecnología e innovación, 2012)

\section{INTRODUCCIÓN}

La producción de conocimientos científicos y tecnológicos en México ha sido un propósito constante en las políticas enfocadas en la educación superior de los últimos años. Con ello se ha intentado incrementar el apoyo financiero a las universidades públicas, así como a otras instituciones de educación superior (IES) y centros públicos de investigación para que fortalezcan sus actividades de investigación científica, tecnológica y de innovación. También se han establecido programas que incentivan la realización de dichas actividades entre su personal de tiempo completo.

No obstante, los esfuerzos emprendidos por ampliar y consolidar un sistema científico, tecnológico y de innovación, hasta ahora no han tenido el éxito esperado pues el aparato de ciencia, tecnología e innovación (CTI) en México sigue siendo todavía incipiente, tal como lo señalan diversos indicadores. El tamaño de la comunidad dedicada a la realización de actividades de investigación e innovación es muy pequeño; su contribución al conocimiento a través de la producción de artículos en revistas con impacto y el registro de patentes es también reducido. Asimismo, la inversión en términos del gasto público y privado en los últimos años es menor al 0.5 por ciento $(0.5 \%)$ del PIB, cifra muy inferior al de las economías más dinámicas del mundo.

Por ello, las universidades públicas, los centros de investigación y otros actores importantes en la esfera de la CTI, han propuesto actualmente una nueva agenda que ponga estas actividades como una de las principales prioridades del nuevo gobierno, a fin de que México pueda contar con las contribuciones de la CTI al desarrollo nacional y, así, poder resolver los grandes problemas de desigualdad social y atraso económico. De esta manera, el país 
podrá conseguir ocupar un lugar destacado entre las naciones más avanzadas del planeta.

En este artículo, presentamos, en primer lugar, una serie de consideraciones sobre las políticas de educación superior en América Latina y en México llevadas a cabo desde la década de los 80 . En esta parte del trabajo, se puede apreciar que una de las principales características fue la enorme expansión de este nivel educativo, en una especie de convergencia con las tendencias mundiales. Dentro del segundo apartado, se examinan algunas de las potenciales contribuciones de la educación superior al desarrollo de la sociedad, aunque también se señalan varias de las limitaciones provocadas por la falta de recursos humanos y financieros que impiden una contribución más significativa a los problemas más acuciantes de la actualidad. El tercer apartado de este trabajo se enfoca a analizar de manera muy amplia las políticas educativas de nivel superior en México, haciendo énfasis en sus resultados. Finalmente, en la última parte se revisan y discuten algunos de los más importantes indicadores del desarrollo científico, tecnológico y de innovación del país. Se incluyen también algunas conclusiones derivadas de los diversos apartados que componen el artículo.

\section{PolítICAS DE EXPANSIÓN DE LA EDUCACIÓN SUPERIOR EN EL MUNDO}

Sin ninguna duda, la expansión del sistema de educación superior constituye el fenómeno más notable de las últimas tres décadas. El sistema de educación superior (SES) en el mundo, entendido como el conjunto de instituciones educativas de nivel superior (conforme a la Clasificación Internacional Normalizada de la Educación -CINE- establecida por la UNESCO') existentes en los diversos países y territorios del orbe, ha vivido una gran expansión en las últimas décadas, multiplicando su matrícula casi trece veces en los últimos 50 años, al pasar de 13 millones de estudiantes en 1960 a 165 millones en el año 2009, y -de acuerdo a los datos disponibles-duplicando su planta académica en los últimos 25 años, al pasar de 4.3 millones de académicos en 1985 a 10.4 millones en 2009 (UNESCO, 2011c) (UNESCO, 2011a) (Jiménez, 2010). Sin embargo, dicho crecimiento no ha sido uniforme ni en el tiempo ni en las distintas regiones y países, de modo que algunas regiones como Asia Oriental y el Pacífico o América del Norte y Europa Occidental han crecido considerablemente, mientras que regiones como África Subsahariana y Asia Central se han expandido mucho menos (UNESCO, 1998) (UNESCO, 2011c); en el caso de la región de América Latina y el Caribe $(\mathrm{ALyC})$, esta ha tenido un crecimiento medio, al haber incrementado su matrícula en 13.3 millones de lugares en el período 1985-2009 (cifra similar a 
la de América del Norte y Europa), frente a los 3.9 millones de lugares creados en la región de África Subsahariana (crecimiento bajo) y los 40.4 millones de lugares generados en la región de Asia Oriental y el Pacífico en el mismo período (crecimiento alto) (UNESCO, 1998) (UNESCO, 2011c). Conviene tener presente que, de manera casi simultánea a la expansión, se dio también un proceso de diversificación institucional. Se crearon, a lo largo de las últimas décadas, diversas instituciones de educación superior como universidades tecnológicas, politécnicas, interculturales e indígenas, así como institutos técnicos y tecnológicos, entre otros.

Dentro del contexto latinoamericano-caribeño, la expansión de los SES nacionales ha sido bastante heterogénea, en términos absolutos, considerando la magnitud de las matrículas nacionales, agrupándose en tres tipos de sistema: un SES de gran expansión (Brasil, México, Argentina, Venezuela), con más de un millón de alumnos matriculados, un SES de mediana expansión (Cuba, Perú, Chile, Ecuador, Bolivia, R. Dominicana), con menos de un millón y más de 250,000 estudiantes y un SES de baja expansión (Guatemala, Paraguay, Uruguay, Honduras, El Salvador, Panamá, Costa Rica, Nicaragua, Haití etc), con menos de 250,000 discentes (UNESCO, 2011c).

Sin embargo, en términos relativos, la expansión diferenciada de los SES nacionales latinoamericanos, considerando las tasas de acceso poblacional a la educación superior, conduce a otro tipo de agrupación: SES en proceso de universalización de la educación superior (Cuba, Venezuela, Argentina, Uruguay, Chile), con tasas de $50 \%$ o más de acceso, SES de cobertura mediana (Panamá, Ecuador, Bolivia, Colombia, Brasil, Perú, R. Dominicana, Paraguay, México), con tasas menores a $50 \%$ y mayores a $25 \%$, y SES de baja cobertura (El Salvador, Costa Rica, Honduras, Guatemala, Nicaragua, Haití), con tasas de $25 \%$ o inferiores (UNESCO, 2011c).

Desde una perspectiva comparada, los SES de México y Brasil, como se puede observar, son sistemas de gran expansión en términos absolutos y de cobertura media en términos relativos. Ambos sistemas son los más multitudinarios de la región latinoamericana, con 6.4 millones y 3 millones de estudiantes respectivamente (casi 10 millones), con datos oficiales de 2010, seguidos por Argentina y Venezuela, con 2.3 y 2.1 millones respectivamente (con datos de 2009) (INEP, 2010) (Calderón, 2010; 2011) (UNESCO, 2011c). De acuerdo con datos del Compendio Mundial de la Educación 2011, editado por la UNESCO (2011c), la matrícula del SES brasileño y del SES mexicano representaban, respectivamente, el $31 \%$ y el $14 \%$ de la matrícula total de ALyC en 2009 , sumando en conjunto casi la mitad de la misma (45\%). 


\section{EDUCACIÓN SUPERIOR Y DESARROLLO}

La contribución de las universidades públicas al desarrollo de América Latina engloba un amplia gama de funciones de carácter social, cultural y político, que no puede ser evaluado sólo en términos de su impacto económico.

Las universidades públicas latinoamericanas cumplen un papel fundamental de preservación y expresión de nuestra cultura y herencia histórica, lo cual es muy importante en la era de la globalización.

El crecimiento económico de América Latina requiere de inversión y aplicación del progreso científico y tecnológico para modernizar los procesos productivos de la región. Para ello, se deben expandir y mejorar tres elementos claves de los sistemas de innovación de la región: 1) la infraestructura científica; 2) la oferta de personal altamente calificado; y 3) una estrecha y funcional vinculación entre los centros de investigación y las empresas productivas.

En América Latina (a diferencia de las naciones desarrolladas), los vínculos entre las universidades y el sector productivo son débiles, con escasa relación entre la agenda de investigación de las universidades y, por parte de las empresas, las necesidades, las presiones técnicas para reducir costos o para innovar.

La literatura, en materia de crecimiento económico, reconoce la relevancia de la formación de capital humano y del avance tecnológico para el desarrollo. La educación superior y la investigación científica son ingredientes esenciales de una economía dinámica e internacionalmente competitiva. (Moreno-Brid y Ruiz-Nápoles, 2009)

Para ampliar y orientar las relaciones entre la investigación universitaria y la actividad industrial, se necesita de un Sistema Nacional de Innovación que comprenda tres factores esenciales:

- Recursos humanos (personal técnico y de investigación).

- Infraestructura adecuada (laboratorios, talleres, equipo de cómputo, bibliotecas etc).

- Instituciones que vinculen a los grupos de investigación de las universidades con las empresas que producen bienes y servicios para el mercado.

Sin una estrategia de largo plazo en la cual tanto el Estado como el sector privado se comprometan a promover la innovación, no será posible que AL logre un auge significativo y persistente en su productividad. Conviene destacar también que uno de los mayores obstáculos para el desarrollo 
tecnológico de la industria local en la región, sigue siendo el hecho de que la mayor parte de las grandes empresas transnacionales que funcionan en América Latina prefieren la tecnología que su propio sistema nacional de innovación produce y no aquella que podrían ofrecerles sus contrapartes locales.

\section{Políticas de la educación superior en México y sus resultados}

El poco promisor panorama de la economía nacional de las últimas tres décadas, demuestra que aun cuando el gobierno mexicano ha aplicado el modelo neoliberal en casi todos los ámbitos de la democracia (muy a su modo) y accedido a la globalización, los efectos que se esperaban no han llegado -o por lo menos no han sido los mismos para todos. En la educación superior, las políticas orientadas en este sentido y con un fuerte apego a las propuestas de los organismos multilaterales, si bien han dinamizado el sistema, han traído consigo diversos efectos, algunos de ellos, incluso, no deseados. Desde el gobierno de Carlos Salinas a la fecha, los ejes que han articulado las políticas de educación superior son la calidad, la cobertura, la equidad y la reforma a la gestión (López Zárate et al, 2011), teniendo como ejes transversales la planeación y la evaluación. Se observa con todo eso, un endurecimiento de los mecanismos de control del Estado sobre las IES.

En materia financiera, se observa una menor participación en los subsidios ordinarios, el incremento en los montos de los fondos extraordinarios por concurso sujetos a resultados de evaluación de desempeño, la promoción de la diversificación de las fuentes de financiamiento, entre las que se incluye el alza de matrículas y los contratos por proyecto. En cuanto a la cobertura y equidad, se observa la diversificación de instituciones, la ampliación de la cobertura, el crecimiento de la matricula, y la promoción de las becas. En lo que corresponde al currículum, se promueve la innovación curricular, la flexibilidad, las salidas intermedias y la formación por competencias, así como el aprendizaje a lo largo de la vida.

En cuanto a la modernización del sistema de gestión, se observan esfuerzos por ampliar la descentralización, por la aplicación de principios de la nueva administración pública, la planeación estratégica, la rendición de cuentas y se exige mayor transparencia en el manejo de los recursos. En lo que se refiere a la evaluación como una política transversal, se ha observado la creación de instancias evaluadoras de instituciones, programas, personal académico y estudiantes. Finalmente, encontramos políticas que promueven la vinculación universidad-empresa, sociedad-universidad y la internacionalización. 
¿Cuáles han sido los resultados para las IES? estos han sido de orden diverso e incluso contradictorio entre sí. Por un lado, instituciones como la UNAM han alcanzado prestigio internacional, por otro, encontramos un desarrollo de ciertas áreas de las IES que han sido estratégicamente impulsadas por lo que han alcanzado competitividad a nivel global, entre estas se encuentran centros de investigación, laboratorios, bibliotecas, programas académicos actualizados y académicos insertos en importantes comunidades de investigación y que publican en revistas de prestigio internacional. Por otra parte, la manera como se concibe la evaluación, la ha reducido a los indicadores y su incremento y, por ello, la ha situado más en los productos que en el proceso. Los indicadores pierden el sentido y la expresión de los cambios que se suceden en el sistema, colocando una mirada obtusa en la educación superior, instrumental y pragmática.

Asimismo, se ha adoptado un nuevo lenguaje administrativista, abandonando el que es propio de la academia, asumiendo términos como planeación estratégica, evaluación, acreditación, certificación de procesos administrativos, dependencias de educación superior, cuerpos académicos, perfil deseable, competitividad académica, transparencia, rendición de cuentas "...los nuevos lenguajes y prácticas han cruzado el tejido universitario y encontrado en los rectores sus principales impulsores al interior de sus comunidades" (López Zárate et al. 2011:1034). Se observan también tendencias hacia la privatización dentro de la educación superior en diferentes niveles y procesos. Ese es el caso del Programa de Financiamiento a la Educación Superior, anunciado a principios de este año por el Presidente de la República.

Existe, además, una academia desarticulada y desorganizada (con sindicatos poco representativos, pérdida de prestaciones sociales y escasa capacidad convocatoria para participar en la transformación social). Esa comunidad académica, sumida en la dinámica de la productividad, en los indicadores y estímulos, en la evaluación y burocratización de sus funciones, tiene poca creatividad para generar un metarrelato alternativo lo suficientemente poderoso para revertir o cambiar el estado actual de la sociedad en general y de la universidad en particular, de manera a transformarse en una lucha contrahegemónica que permita recuperar el conocimiento como un bien intangible al que todos tienen derecho. La comunidad estudiantil es mayoritariamente apática, escasamente comprometida con la transformación de la sociedad, demandante de un conocimiento instrumental, con escaso interés por los asuntos políticos.

En la universidad también son cada vez más notorias las diferencias entre los beneficios que se recogen como producto de los capitales social, 
cultural y económico. Existen amplias brechas entre los salarios de los académicos de tiempo completo (debido a los programas de estímulos) y los profesores de asignatura; entre quienes tienen estudios de posgrado y aquellos que no; entre quienes estudian en una universidad local y los que lo hacen en una foránea; entre quienes hablan un solo idioma y los hablan dos o más; entre disciplinas notoriamente apoyadas e importantes (por el gobierno o las autoridades) y las que tienden a valorarse poco y a ser cada vez menos atendidas; entre los servicios que reciben quienes pueden pagar y los que no (un notorio ejemplo de ello es el acceso a los alimentos que cada quien puede comprar dentro de la universidad). El papel que en este sentido ha cumplido la universidad ha sido el de la reproducción de las diferencias de la sociedad y del orden establecido.

INVESTIGACIÓN CIENTíFICA, TECNOLÓGICA E INNOVACIÓN EN MÉXICO

Las actividades científicas y tecnológicas en México son escasas y poco relevantes, desde el punto de vista económico, comparadas con la situación que guardan en la mayoría de los países de la OCDE. Los indicadores disponibles así lo muestran: el gasto federal en ciencia y tecnología (GFCyT) ha tenido un comportamiento variable durante el períodoperíodo 1990-2011, como resultante de las políticas de financiamiento dictadas por distintos gobiernos federales, tanto en relación al Producto Interno Bruto (PIB) ${ }^{1}$ como al Gasto Programable del Sector Público Federal (GPSPF)². En el períodoperíodo 1990-1994, correspondiente al gobierno de Salinas (ver Tabla 1), el gasto científico-tecnológico con respecto al PIB fue de $0.34 \%$ en promedio anual -el más bajo en las dos últimas décadas-; en el períodoperíodo 1995-2000, durante el gobierno de Zedillo, el gasto promedio anual fue de $0.46 \%$-el más alto en ambas décadas-. En el período 2001-2006, en el gobierno de Fox, el gasto promedio anual fue de $0.36 \%$, mientras que en el período 2007-2011, correspondiente al gobierno de Calderón, el gasto promedio fue de $0.38 \%$ (promedios extraídos de los datos de la Tabla 1). Esomuestra el desdén de la élite política con respecto al fortalecimiento de la ciencia y la tecnología, a pesar de los insistentes llamados de la comunidad científica a elevar la inversión científica al 1\% del PIB.

Con relaicón al GPSPF, este fue de $2.08 \%$ anual en promedio en el período correspondiente a Salinas, de $2.54 \%$ en promedio en el gobierno de Zedillo, de $2.23 \%$ en promedio en el de Fox y de $1.99 \%$ en promedio en el Calderón, registrándose el gasto promedio más alto con Zedillo y el más bajo con Calderón (promedios extraídos de la Tabla 1), tendencia a la baja que 
refleja claramente el desinterés estatal en incentivar el desarrollo científico nacional, el cual no ha sido una de sus prioridades.

Tabla 1 - Gasto federal en ciencia y tecnología (GFCyT) - 1990-2011

\begin{tabular}{|c|c|c|c|c|c|c|}
\hline \multirow[t]{2}{*}{$\begin{array}{c}\text { PeríodoPeríodo } \\
\text { gubernamental } \\
\text { sexenal }\end{array}$} & \multirow[t]{2}{*}{ Año } & \multirow{2}{*}{$\begin{array}{c}\text { Gasto } \\
\text { Federal en } \\
\text { Ciencia y } \\
\text { Tecnología } \\
\text { (GFCYT) } \\
\begin{array}{c}\text { Millones } \\
\text { de pesos }\end{array}\end{array}$} & \multicolumn{2}{|c|}{$\begin{array}{l}\text { Producto Interno Bruto } \\
\text { (PIB) }\end{array}$} & \multicolumn{2}{|c|}{$\begin{array}{c}\text { Gasto Programable del } \\
\text { Sector Público Federal } \\
\text { (GPSPF) }\end{array}$} \\
\hline & & & $\begin{array}{l}\text { Millones } \\
\text { de pesos }\end{array}$ & $\begin{array}{l}\text { GFCyT/ } \\
\text { PIB (\%) }\end{array}$ & $\begin{array}{l}\text { Millones } \\
\text { de pesos }\end{array}$ & $\begin{array}{l}\text { GFCyT/ } \\
\text { GPSPF } \\
(\%)\end{array}$ \\
\hline \multirow{5}{*}{$\begin{array}{c}\text { Carlos Salinas de } \\
\text { Gortari (1988- } \\
\text { 1994) }\end{array}$} & 1990 & 2035 & 738898 & 0.28 & 117122 & 1.74 \\
\hline & 1991 & 3156 & 949148 & 0.33 & 148879 & 2.12 \\
\hline & 1992 & 3613 & 1125334 & 0.32 & 178266 & 2.03 \\
\hline & 1993 & 4588 & 1256196 & 0.37 & 206987 & 2.22 \\
\hline & 1994 & 5766 & 1420159 & 0.41 & 249481 & 2.31 \\
\hline \multirow{6}{*}{$\begin{array}{l}\text { Ernesto Zedillo } \\
\text { Ponce de León. } \\
\text { (1994-2000) }\end{array}$} & 1995 & 6484 & 1840431 & 0.35 & 290424 & 2.23 \\
\hline & 1996 & 8840 & 2529909 & 0.35 & 403450 & 2.19 \\
\hline & 1997 & 13380 & 3179120 & 0.42 & 528124 & 2.53 \\
\hline & 1998 & 17789 & 3848218 & $\Rightarrow 0.46$ & 600583 & $\Rightarrow 2.96$ \\
\hline & 1999 & 18788 & 4600488 & 0.41 & 711228 & 2.64 \\
\hline & 2000 & 22923 & 5497736 & 0.42 & 855286 & 2.68 \\
\hline \multirow{6}{*}{$\begin{array}{l}\text { Vicente Fox } \\
\text { Quezada } \\
(2000-2006)\end{array}$} & 2001 & 23993 & 5811776 & 0.41 & 937214 & 2.56 \\
\hline & 2002 & 24364 & 6267474 & 0.39 & 1078861 & 2.26 \\
\hline & 2003 & 29309 & 7555803 & 0.39 & 1241853 & 2.36 \\
\hline & 2004 & 27952 & 8561305 & 0.33 & 1326952 & 2.11 \\
\hline & 2005 & 31339 & 9220649 & 0.34 & 1477368 & 2.12 \\
\hline & 2006 & 33276 & 10344065 & 0.32 & 1671175 & 1.99 \\
\hline \multirow{5}{*}{$\begin{array}{c}\text { Felipe Calderón } \\
\text { Hinojosa } \\
(2006-2012)\end{array}$} & 2007 & 35832 & 11290752 & 0.32 & 1911321 & 1.87 \\
\hline & 2008 & 43829 & 12153436 & 0.36 & 2229154 & 1.97 \\
\hline & 2009 & 45974 & 11844514 & 0.39 & 2459610 & 1.87 \\
\hline & 2010 & 54436 & 13075798 & 0.42 & 2640625 & 2.06 \\
\hline & 2011 & 58,810 & $14,352,867$ & 0.41 & $2,686,300$ & 2.19 \\
\hline
\end{tabular}

Notas: Millones de pesos corrientes. Para el períodoperíodo 2000-2004 se incluye el estímulo fiscal a la investigación y desarrollo tecnológico. Para los años 2002 y 2003, cifras revisadas del Sector Energía.

Fuentes: Para 1990-2002: CONACYT. Informe General del Estado de la Ciencia y la Tecnología. México. 2004, 2006-2008.

Para 2003-2010: CONACYT. Informe General del Estado de la Ciencia y la Tecnología. México. 2010.

Para 2011: CONACYT. Indicadores de actividades Científicas y tecnológicas. Edición de bolsillo. México 2011.

Datos disponibles de 1990-2010 en: http://www.inegi.org.mx/Sistemas/temasV2/Default.aspx?s=est\&c=19007 
En lo que se refiere a la proporción del Gasto Interno en Investigación y Desarrollo Experimental con relación al PIB, en el 2008 fue de $0.42 \%$. En una comparación con países de la OCDE, resulta ser de los más bajos, ya que países como Suecia, Japón, Corea, Estados Unidos y Alemania, invierten el $3.75 \% ; 3.44 \% ; 3.21 \% ; 2.77 \%$ y $2.5 \%$ del mismo indicador, respectivamente.

Por otro lado, la matrícula de posgrado ha tenido un crecimiento notable, pues en 1980 era de apenas 22,502, mientras que en 2010 llegó a 204,275 . Sin embargo, este número representa menos del $10 \%$ de la población total en educación superior $(3,071,643)$. Asimismo, los egresados y graduados en el nivel de posgrado constituyen un elemento potencial para el desarrollo científico y tecnológico del país. Su número pasó de 38 mil en 2002 a 72 mil 622 en 2009. Sin embargo, a pesar de que estuvieron cerca de duplicarse en dicho período, su número todavía es muy pequeño, comparado con los casi tres millones de estudiantes de educación superior. Más aún, en 2009, los estudiantes de doctorado sólo representaron el $6.6 \%$ del total, mientras que los de maestría constituyeron el $63.2 \%$ y los de especialización el $30.2 \%$. La mayor parte de las matrículas de ese año estuvo formada por estudiantes de la ciencias de la salud, de las ciencias sociales y humanidades.

Asimismo, el número de graduados en programas de doctorado se ha incrementado significativamente en los últimos años (ver Tabla 2) como resultado del aumento en la matrícula de educación superior en general y de la matrícula del posgrado en lo particular, de modo que, entre 2000 y 2010 , prácticamente se triplicó el número de egresados, pasando de una proporción de 11 doctorados por millón de habitantes en 2000 a una proporción de 26 doctorados por millón en 2010 (ver el Total en la Tabla 2). En lo que se refiere a las áreas científicas, los graduados de doctorado por millón de habitantes en Ciencias Naturales se duplicaron en el período 2000-2010, mientras que los de Ciencias Sociales casi se triplicaron, destacando el crecimiento del número de graduados tanto en Ciencias sociales y administrativas como en Ingeniería y tecnología (ver Tabla 2). No obstante, hay que señalar que el número de doctores graduados anualmente en México (2,600 aproximadamente), aún es muy pequeño comparado con países de la región, como Brasil, que gradúan al año más de 11 mil doctores.

Además del número de estudiantes de posgrado, otro indicador lo constituye el número de becas vigentes del Consejo Nacional de Ciencia y Tecnología (CONACYT). Su número se incrementó de 10,249 en 2000 a 30,634. En el período 2000-2009 las becas nacionales aumentaron de 7,918 a 28,910 y, a su vez, las becas al extranjero en ese mismo período apenas crecieron de 2,331 a 2,424. Cabe señalar que las nacionales fueron en su 


\begin{tabular}{|c|c|c|c|c|c|c|c|c|c|c|c|c|c|c|c|c|c|c|}
\hline $\begin{array}{l}\grave{N} \\
\stackrel{\vdots}{2}\end{array}$ & & & 옹 & in & $£$ & 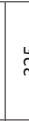 & & 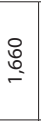 & $\begin{array}{l}\infty \\
\underset{+}{+}\end{array}$ & & $\stackrel{n}{\curvearrowright}$ & $\stackrel{\dddot{m}}{\sigma}$ & 足 & $\stackrel{\text { I }}{=}$ & $\begin{array}{l}\infty \\
\stackrel{\infty}{i}\end{array}$ & 迥 & 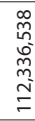 & 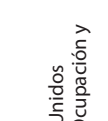 \\
\hline ઠे & & & î & 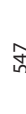 & $\overline{7}$ & & & 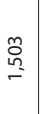 & $\begin{array}{l}\stackrel{0}{\dot{j}} \\
\end{array}$ & & f & $\stackrel{+}{\sigma}$ & $\underset{\mathbb{N}}{\mathbb{N}}$ & $\stackrel{m}{=}$ & 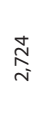 & $\stackrel{m}{\omega}^{m}$ & $\begin{array}{l}\text { 음 } \\
\text { 员 } \\
\text { م. }\end{array}$ & 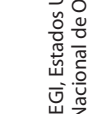 \\
\hline : & & & î̀ & $\stackrel{\leftrightarrow}{\stackrel{\infty}{\sigma}}$ & ๑ & & & $\underset{J}{J}$ & $\stackrel{\stackrel{m}{m}}{\stackrel{m}{m}}$ & & : & $\stackrel{\infty}{\text { f }}$ & $\stackrel{\circ}{\Xi}$ & $\stackrel{\text { ¿̊ }}{\circ}$ & $\begin{array}{l}\text { 岕 } \\
\text { N }\end{array}$ & ભે & $\begin{array}{l}\infty \\
i n \\
0 \\
0 \\
0 \\
\stackrel{0}{0} \\
0\end{array}$ & 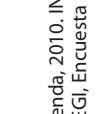 \\
\hline ڤ్ & & & $\sum^{m}$ & F & 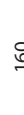 & & & 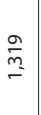 & 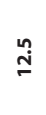 & & 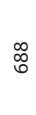 & $\stackrel{\circlearrowright}{\sim}$ & ઠ̊ & $\bar{\sigma}$ & 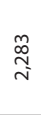 & 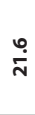 & 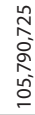 & 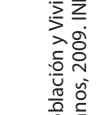 \\
\hline ¿ัర & & & 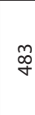 & 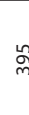 & 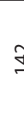 & & & $\stackrel{\sigma}{m}$ & 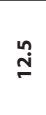 & & $\stackrel{\infty}{\Re}$ & $\stackrel{\circ}{\sim}$ & $\stackrel{\infty}{\curvearrowright}$ & $\stackrel{\circ}{r}$ & $\underset{\sim}{\mathfrak{i}}$ & $\overline{\text { ஸे }}$ & 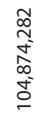 & 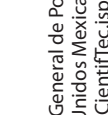 \\
\hline : & & & $\underset{g}{q}$ & $\stackrel{9}{\text { I }}$ & $\stackrel{9}{\vdots}$ & 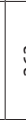 & & 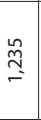 & $\stackrel{\stackrel{Q}{g}}{=}$ & & $\stackrel{\sigma}{\sigma}$ & $\stackrel{m}{\sim}$ & $\hat{\kappa}$ & นุ & 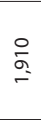 & $\stackrel{+}{\infty}$ & 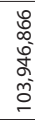 & 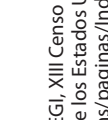 \\
\hline ఫั & & & f & $\stackrel{n}{n}$ & $\tilde{n}$ & 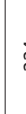 & & $\begin{array}{l}\text { on } \\
\stackrel{0}{0} \\
-\end{array}$ & $\stackrel{m}{\stackrel{m}{0}}$ & & $\frac{\sigma}{\sigma}$ & $\bar{\sim}$ & రి & : & $\stackrel{\infty}{0}$ & $\stackrel{m}{\circ}$ & $\begin{array}{l}\overline{1} \\
\infty \\
\overline{0} \\
0 \\
0 \\
0\end{array}$ & 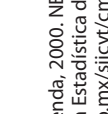 \\
\hline 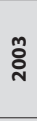 & & & $\bar{m}$ & $\stackrel{\infty}{\approx}$ & $\stackrel{g}{\tilde{m}}$ & 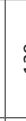 & & $\begin{array}{c}\stackrel{\infty}{\infty} \\
\infty\end{array}$ & $\hat{\infty}$ & & 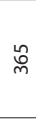 & $\stackrel{\widetilde{\sigma}}{\sigma}$ & กิ & กี & $\stackrel{g}{\underset{\sigma}{f}}$ & $\stackrel{\stackrel{g}{m}}{\sim}$ & $\begin{array}{l}\infty \\
0 \\
\alpha \\
\alpha \\
\alpha \\
\vdots \\
\end{array}$ & 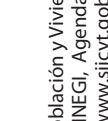 \\
\hline ర్ & & & $\stackrel{\mathscr{D}}{m}$ & g & 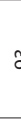 & & & $\underset{\infty}{\tilde{\infty}}$ & $\underset{\infty}{\mathbf{\infty}}$ & & 今 & 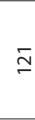 & $\stackrel{n}{\sigma}$ & $\bar{f}$ & $\stackrel{\infty}{\stackrel{\infty}{\sim}}$ & 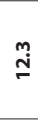 & $\begin{array}{l}\text { m. } \\
\infty \\
\mathrm{o}_{2} \\
\stackrel{\circ}{\circ} \\
\stackrel{0}{0}\end{array}$ & 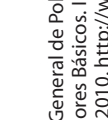 \\
\hline ¿্ं & & & $\bar{m}$ & ำ & $\Phi$ & & & ¿ & $\bar{r}$ & & 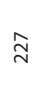 & $\underset{f}{\ddagger}$ & in & $\hat{m}$ & 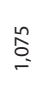 & $\stackrel{\infty}{\stackrel{0}{\circ}}$ & $\begin{array}{l}\frac{1}{n} \\
\kappa \\
\kappa \\
\alpha \\
\alpha\end{array}$ & 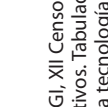 \\
\hline : & & & $\stackrel{\infty}{\sim}$ & $\stackrel{m}{m}$ & 3 & 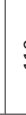 & & 잉 & 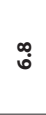 & & $\stackrel{\bar{\infty}}{\sim}$ & $\stackrel{\mathscr{N}}{\stackrel{2}{2}}$ & ఫ্ণ & $\bar{f}$ & $\stackrel{\circ}{\circ}$ & $\stackrel{ }{\circ}$ & $\begin{array}{l}m \\
\hat{n} \\
\infty \\
\infty \\
m \\
\infty \\
\infty\end{array}$ & 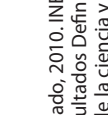 \\
\hline & 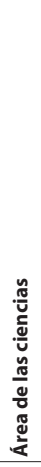 & 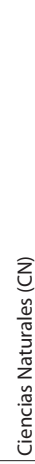 & 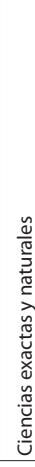 & 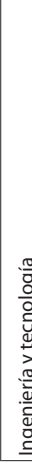 & 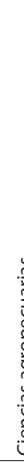 & & & 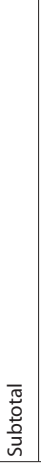 & 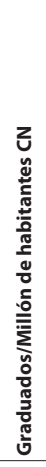 & 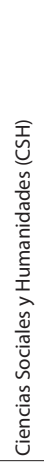 & 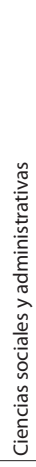 & 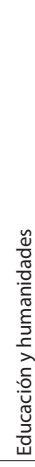 & $\begin{array}{l}\bar{\pi} \\
0 \\
0 \\
0 \\
\text { ज. }\end{array}$ & 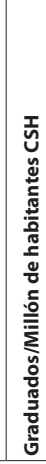 & $\begin{array}{l}\overline{\text { s。 }} \\
\stackrel{\circ}{\circ}\end{array}$ & 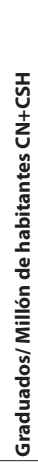 & 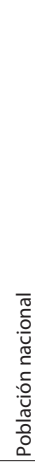 & 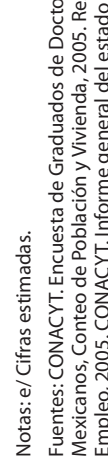 \\
\hline
\end{tabular}


mayoría para el nivel de maestría $(17,241)$, mientras que las del extranjero fueron sustancialmente mayores para el nivel de doctorado $(2,017)$. El mayor porcentaje de estas últimas fue para estudios en los Estados Unidos (25\%), el Reino Unido (23.4\%), España (21.4\%), Alemania (8.3\%), Francia (6.8\%) y Canadá (5.8\%) (CONACYT, 2009).

El número de investigadores por cada 1,000 personas de la población económicamente activa también es muy pequeño. En 2007, el porcentaje fue de $0.88 \%$, habiendo sido de $0.57 \%$ en 2000 , lo que denota un crecimiento apenas perceptible. Ese indicador contrasta fuertemente cuando comparado con los de países como Japón (10.3\%), Corea (9.7\%), Estados Unidos (9.2\%), Canadá (7.9\%), Francia (7.6\%) y Alemania (7.2\%), e incluso con Argentina (2.0\%).

El comportamiento del Sistema Nacional de Investigadores (SNI) -que aglutina a la élite de investigadores científicos en México- ha sido variable desde su fundación en 1984, con ritmos de crecimiento y decrecimiento diferenciados (ver gráfica 1), en función de políticas también diferenciadas, de tal manera que, después de un ciclo virtuoso de alto crecimiento entre 1984 y 1992 (con un crecimiento acumulado de 373\%), en 1992-2000 entra a una fase de decrecimiento y desaceleración del S.N.I. (con un crecimiento acumulado mínimo de sólo 13\%), mientras que en los períodos 2000-2008 y 2003-2011 se da una relativa recuperación (crecimiento acumulado regular de $97 \%$ y $92 \%$, respectivamente). El mayor porcentaje de investigadores pertenecientes al SNI, en 2009, se concentra en el área de biología y química (17.4\%), seguido de físico-matemáticas y ciencias de la tierra (17.4\%), humanidades y ciencias de la conducta (16.1\%), ciencias sociales (15.9\%), ingeniería (14.4\%), biotecnología y ciencias agropecuarias (11.1\%), y medicina y ciencias de la salud (9.3\%).

La producción científica nacional, referida a la publicación de artículos científicos en revistas especializadas reconocidas internacionalmente (CONACYT, 2010: 91-111), ha aumentado significativamente en las últimas tres décadas, al transitar de 4,894 artículos en el quinquenio 1981-1985 a 43,519 artículos en el quinquenio 2006-2010 (casi diez veces más) -ver Tabla 3-, asimismo, en el ámbito latinoamericano, México pasa del tercer lugar al segundo -debajo de Brasil- en producción de artículos, superando a Argentina; no obstante lo anterior, la distancia de México respecto a Brasil aumentó en los tres decenios (de $124 \%$ a $234 \%$, entre 1981-1985 y 2011). Por otra parte, en el plano internacional, la participación de México en la producción mundial de artículos se triplica, al pasar del $0.21 \%$ al $0.84 \%$ entre 1981 y 2011 (datos calculados a partir de la Tabla 3), manteniendo su posición mundial (alrededor de la posición 16) con respecto a la mayoría de 


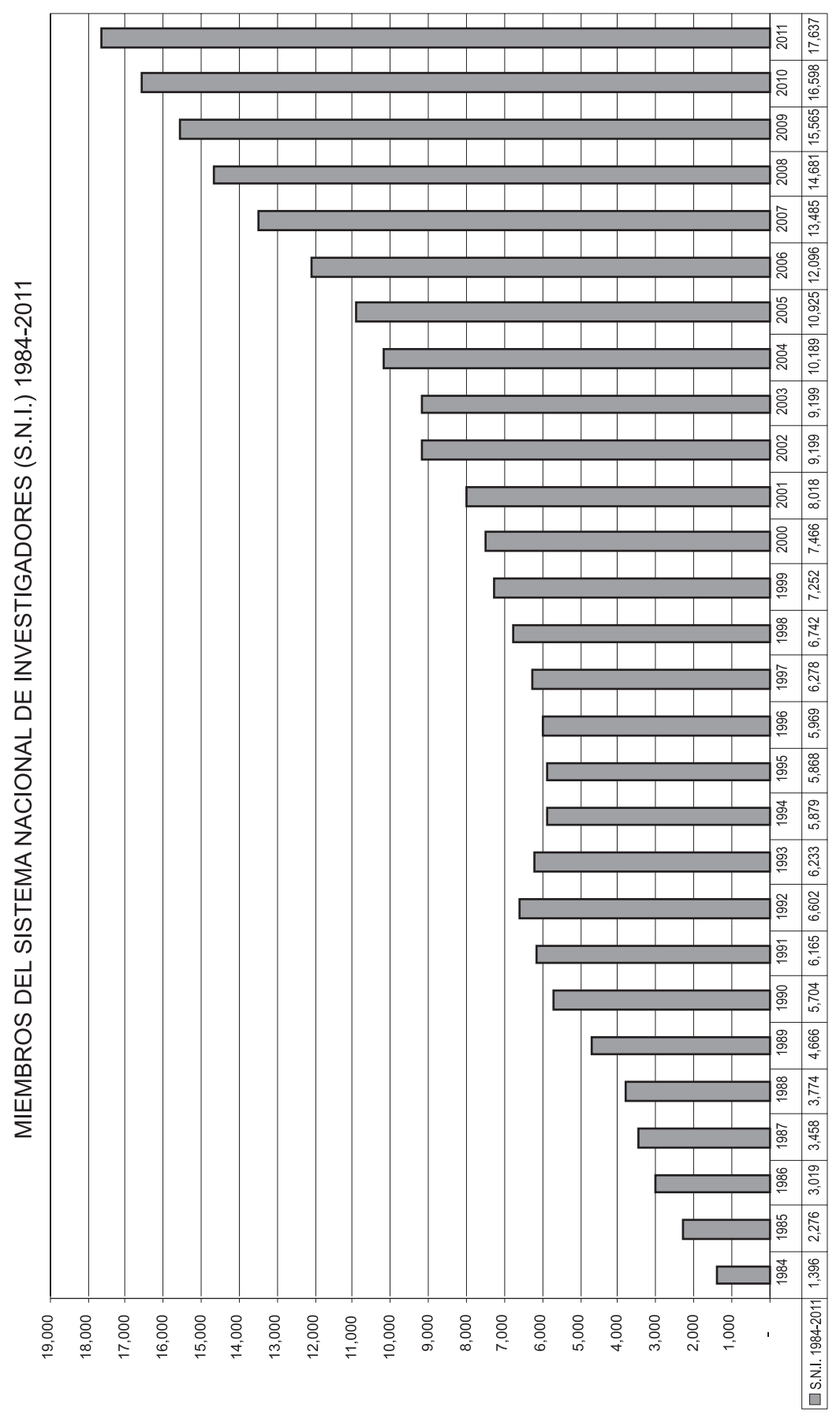


países productores (exceptuando el caso de Turquía, que, como resultado de políticas diferenciadas, a partir del quinquenio 1996-2000, supera a México, después de haber estado abajo durante varios quinquenios). Sin embargo, comparativamente, la participación de México es muy reducida en el concierto internacional, considerando que los mayores porcentajes de producción pertenecen a Estados Unidos, China, Reino Unido, Alemania y Japón (ver Tabla 3).

El llamado coeficiente de inventiva (número de solicitudes de patentes de residentes nacionales por cada 10 mil habitantes) en México, como un indicador de la capacidad nacional de generación de nuevo conocimiento aplicado en el mundo, demuestra una clara tendencia a la baja en el período 1980-2011, con altibajos entre 1980 y 1990, un claro y prolongado decaimiento entre 1991 y 2008 y una relativa recuperación a partir de 2009, pero sin llegar a los niveles alcanzados en los ochentas (ver Gráfica 2). Del mismo modo, el número de patentes solicitadas y concedidas en México muestra que en 2008 sólo se solicitaron 685 por mexicanos y 15,896 por extranjeros, de los cuales se concedieron 197 y 10,243 respectivamente. Cuando se compara este indicador a nivel internacional, se traduce una alta relación de dependencia (24.90) y muy bajas tasas de difusión (0.71) y coeficiente de inventiva $(0.06)$.

En síntesis y con base en estos y otros indicadores, puede decirse que entre los principales obstáculos al desarrollo científico y tecnológico, así como para la innovación en América Latina, se encuentran los siguientes:

- Muy baja contribución a la producción científica mundial (1.5\%).

- Escaso número de científicos y tecnólogos (85 mil en Brasil, 645 en Ecuador).

- Bajos montos de inversión en CyT por parte de los gobiernos (menos del $0.5 \%$ del PIB en muchos países, incluido México) y las empresas privadas que casi no realizan actividades de investigación y desarrollo (I\&D).

- La mayor parte del gasto en CyT es realizado por el Estado. En los países desarrollados sucede lo contrario: son las empresas privadas las que gastan más.

- La distribución del gasto en CyT no se dirige a la ingeniería en sus diversas áreas.

- Falta interacción y colaboración entre investigadores y de ellos con la industria (importancia de la tecnología). 
Tabla 3 - Artículos publicados por países seleccionados (agrupación quinquenal) 1981-2011

\begin{tabular}{|c|c|c|c|c|c|c|c|c|}
\hline & País & 1981-1985 & $1986-1990$ & 1991-1995 & $1996-2000$ & 2001-2005 & 2006-2010/e & 2011/e \\
\hline 1. & E.U.A. & 900,655 & $1,021,832$ & $1,177,544$ & $1,214,575$ & $1,304,895$ & $1,610,266$ & 353,143 \\
\hline 2. & China & sd & sd & sd & 101,152 & 209,774 & 505,454 & 137,220 \\
\hline 3. & $\begin{array}{l}\text { Reino } \\
\text { Unido }\end{array}$ & 202,644 & 226,472 & 277,017 & 326,443 & 345,950 & 431,308 & 95,109 \\
\hline 4. & Alemania & 175,905 & 200,028 & 242,078 & 304,288 & 334,985 & 417,065 & 91,834 \\
\hline 5. & Japón & 150,186 & 198,629 & 265,403 & 328,818 & 360,453 & 386,862 & 78,793 \\
\hline 6. & Francia & 118,973 & 143,653 & 183,420 & 223,528 & 238,859 & 300,764 & 66,829 \\
\hline 7. & Canadá & 105,864 & 131,369 & 157,461 & 160,935 & 178,294 & 255,215 & 59,546 \\
\hline 8. & Italia & 54,675 & 73,059 & 107,034 & 141,796 & 172,880 & 239,422 & 55,105 \\
\hline 9. & España & 22,382 & 38,425 & 64,506 & 96,552 & 124,210 & 197,443 & 47,501 \\
\hline 10. & India & sd & sd & sd & 75,933 & 98,277 & 177,616 & 43,041 \\
\hline 11. & Corea & sd & sd & sd & 47,830 & 91,664 & 169,647 & 42,549 \\
\hline 12. & Brasil & 10,963 & 14,469 & 23,287 & 39,149 & 63,737 & 133,829 & 34,863 \\
\hline 13. & Turquía & 1,907 & 3,653 & 8,952 & 20,930 & 49,195 & 96,536 & 24,969 \\
\hline 14. & Polonia & 22,139 & 26,170 & 30,767 & 41,140 & 56,908 & 86,833 & 20,600 \\
\hline 15. & Grecia & 5,583 & 8,734 & 13,713 & 20,568 & 30,466 & 49,698 & 11,265 \\
\hline 16. & México & 4,894 & 6,681 & 11,210 & 19,929 & 28,713 & 43,519 & 10,449 \\
\hline 17. & Portugal & 1,530 & 3,107 & 6,161 & 12,014 & 20,551 & 38,285 & 9,353 \\
\hline 18. & Argentina & 6,258 & 8,954 & 11,028 & 18,268 & 23,154 & 32,136 & 7,427 \\
\hline 19. & Chile & 3,596 & 4,695 & 6,197 & 8,106 & 11,936 & 20,216 & 4,996 \\
\hline 20. & Colombia & 633 & 782 & 1,165 & 2,349 & 3,613 & 8,961 & 2,540 \\
\hline 21. & Venezuela & 1,999 & 2,143 & 2,932 & 3,953 & 4,731 & 6,279 & 1,429 \\
\hline & Otros & 495,568 & 525,339 & 494,220 & 296,378 & 167,011 & 235,742 & 51,952 \\
\hline \multicolumn{2}{|c|}{ Total Mundial } & $2,286,354$ & $2,638,194$ & $3,084,095$ & $3,504,634$ & $3,920,256$ & $5,443,095$ & $1,250,512$ \\
\hline
\end{tabular}

Notas: /e: datos estimados.

Fuentes: Institute for Scientific Information, 1997-2010. CONACYT. Indicadores de Actividades Científicas y Tecnológicas 1997. CONACYT. Anexo estadístico del Informe general del estado de la ciencia y la tecnología, 2007. CONACYT. Anexo estadístico del Informe general del estado de la ciencia y la tecnología, 2010. CONACYT. Indicadores de actividades científicas y tecnológicas. Edición de bolsillo. México 2011. http://www.siicyt.gob. $\mathrm{mx} / \mathrm{siicyt} / \mathrm{cms} /$ paginas/InfoGralEstCyT0207.jsp 


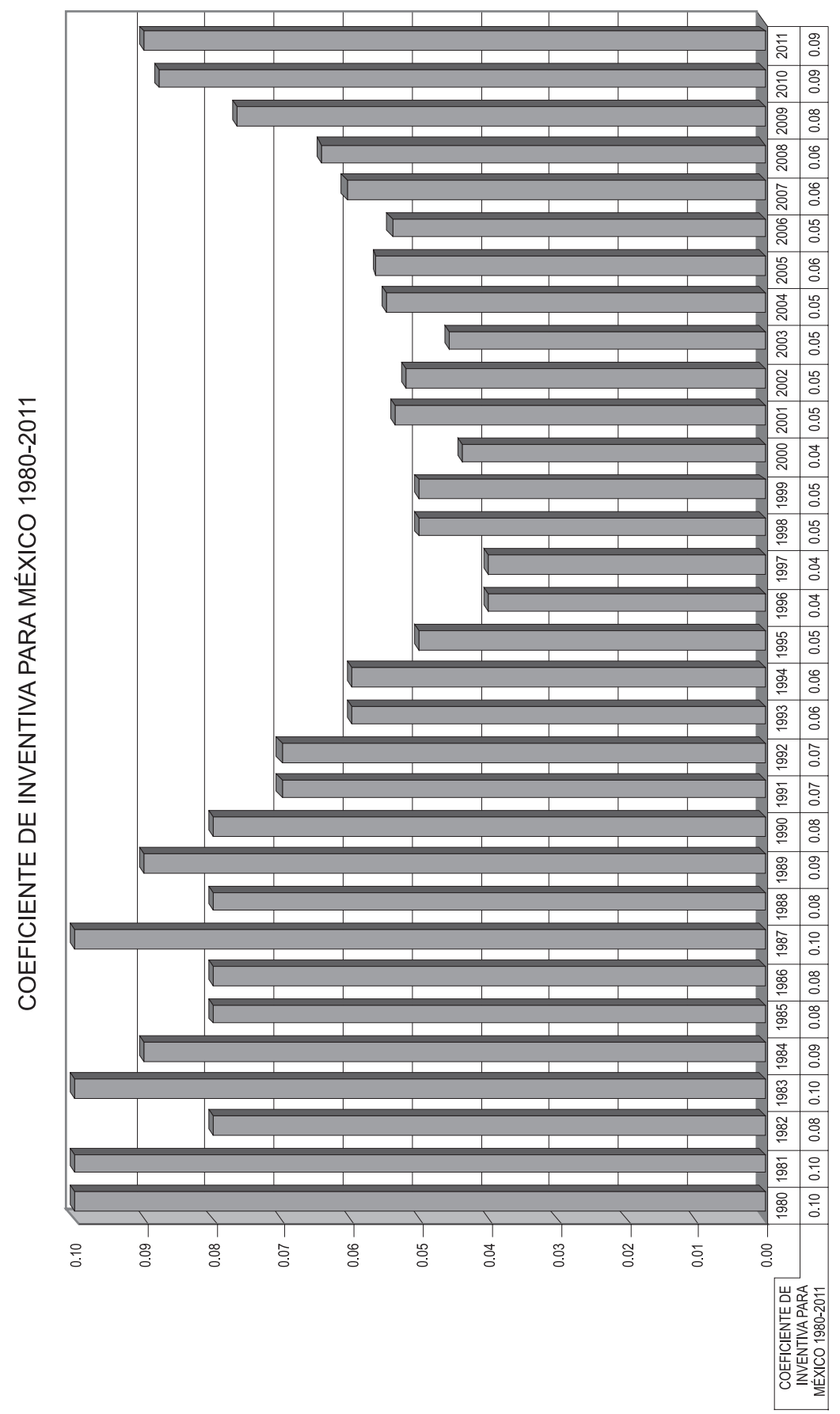




\section{Conclusiones}

El panorama planteado en este trabajo intentó mostrar la situación de algunas de principales políticas de educación superior, ciencia, tecnología e innovación que han sido desarrolladas en América Latina, en lo general, y en México, en lo particular. De esta manera, se subrayó que uno de los procesos que le ha dado mayor dinamismo ha sido el de la expansión, a la que ha acompañado casi desde su inicio, la diversificación institucional. También se examinaron varias de las condiciones que han de tener las universidades y la IES para constituirse en motores del cambio y del desarrollo social y económico de los países. Se tomó como referencia nuevamente el caso de México y se plantearon algunos de los desafíos más importantes que las instituciones educativas y de investigación deben superar para alcanzar su pleno desarrollo e insertarse de lleno en la llamada sociedad del conocimiento. En la parte final del artículo, se revisaron las condiciones en las que se realizan las actividades de investigación científica, tecnológica y de innovación en México, destacándose las condiciones desventajosas y la serie de carencias y limitaciones que las aquejan.

Frente a ese difícil y complejo panorama, cobra una gran importancia la iniciativa muy reciente encabezada por los rectores y directores generales de las principales universidades, centros de investigación y diversas IES. Ese emprendimiento pugna por establecer una agenda nacional en materia de ciencia, tecnología e innovación (CTI), "que permita hacer del conocimiento y la innovación una palanca fundamental para el crecimiento económico sustentable del país, que favorezca el desarrollo humano, posibilite una mayor justicia social, consolide la democracia y la paz, y fortalezca la soberanía nacional" (Hacia una agenda nacional de Ciencia, Tecnología e Innovación, 2012). Planteado en un horizonte de 25 años, el documento propone al nuevo gobierno mexicano no solamente el financiamiento creciente de las actividades $\mathrm{CTI}$, sino el apuntalamiento institucional, dentro de la estructura gubernamental, de un instrumento de coordinación y promoción de la ciencia, la tecnología y la innovación.

Si prosperara ese planteamiento colectivo de la comunidad científica y tecnológica del país, México se pondría, en corto plazo, en condiciones de insertarse en la llamada sociedad del conocimiento, pero, aún más, sería capaz de traducir los beneficios de tal inserción en el mejoramiento de las condiciones de vida de sus habitantes, sobre todo de aquellos con mayores necesidades. De no ser así, se correrá el riesgo de que el país quede aún más rezagado frente a las economías más dinámicas del planeta, poniéndose en 
peligro no sólo el bienestar de grandes sectores de la población, sino también su viabilidad como nación libre y soberana.

HIGHER EDUCATION POLICIES ANDTHE PRODUCTION OF KNOWLEDGE IN MEXICO (1980-2011)

ABSTRACT: This study deals with different processes and indicators related to Higher Education ( $\mathrm{HE}$ ) and the production of knowledge in Mexico over the last thirty years. The data which were analyzed would indicate that despite the rapid expansion of $\mathrm{HE}$ in Mexico from 1980 to 2011, productivity in research and the production of knowledge are very low, not just when compared with more developed countries but also with others in the region. The accelerated expansion of $\mathrm{HE}$ is a global phenomenon, even though more visible in some regions than in others. In comparative terms, Mexico and Brazil showed a marked increase in absolute terms, although their overall levels of coverage are somewhat average. In the case of Mexico, indicators of scientific, technological and innovative capacity showed a low investment in terms of GDP. The number of postgraduates has increased significantly over recent years although they are still few in proportion to the total number. The number of doctoral students who graduate annually is quarter that of Brazil. The research community is greatly reduced, both in global and in regional terms. Despite the significant increase in the number of articles published in international jornals, it is still very low. The coefficient of innovative work, however, tends to decrease. Even though the indicators are insufficient and limited, there is room for moderate optimism because of the increase in the number of students at higher and postgraduate education levels, as well as the perspective of greater governmental investment in research, technology and innovation.

KEYwORDS: Higher Education. Research. Production of Knowledge.

\section{POLÍTICAS DE EDUCAÇÃO SUPERIOR E PRODUÇÃO DE CONHECIMENTOS NO MÉXICO (1980-2011)}

RESUMO: Neste artigo, serão revisados diversos processos e indicadores relacionados com a educação superior (ES) e a produção de conhecimentos no México durante as últimas três décadas. Os dados analisados indicam que, apesar da rápida expansão da ES mexicana durante o período de 1980 a 2011, a produtividade na pesquisa e a produção de conhecimentos fue muito baixa, não só em comparação com as nações mais avançadas, se não com países da região. O crescimento acelerado da ES é um fenômeno mundial, ainda que é mais visível em algumas regiões que em outras. Comparativamente, México e Brasil mostram um forte crescimento em termos absolutos, mas os seus níveis ainda são de grau médio. Os indicadores que relacionam a capacidade científica, tecnológica e de inovação, no caso do México, mostram um baixo investimento em relação ao PIB. Os estudantes de pós graduação aumentaram significativamente nos últimos anos, mas continuam sendo poucos em relação ao 
número total. A quantidade de doutores formados anualmente representa um quarto dos que se formam no Brasil. A pesar do aumento de artigos publicados em revistas internacionais, seu número ainda é pequeno. O coeficiente de criatividade apresenta, além disso, uma tendência à diminuição. Mesmo que os índices sejam insuficientes e limitados, há lugar para o otimismo moderado devido ao crescimento no número de estudantes em ES e pós graduação, assim como às perspectivas de maior investimento governamental em ciência, tecnologia e inovação.

PalavRas-Chave: Educação Superior; Investigação; Produção de Conhecimento.

\section{REFERENCIAS}

ACADEMIA MEXICANA DE CIENCIAS-FORO CONSULTIVO CIENTÍFICOYTECNOLÓGICO. Una reflexión sobre el sistema nacional de investigadores a 20 años de su creación. México: AMC-FCCyTI, Febrero 2005.

CALDERÓN H., Felipe. Cuarto Informe de Gobierno. México: Presidencia de la República, 2010.

CALDERÓN H., Felipe. Quinto Informe de Gobierno. México: Presidencia de la República, 2011.

CONACYT. Indicadores de actividades científicas y tecnológicas. México: SEP-CONACYT, 1996.

CONACYT. Indicadores de actividades científicas y tecnológicas. México: SEP-CONACYT, 1997.

CONACYT. Informe general del estado de la ciencia y la tecnología. México: CONACYT, 2002.

CONACYT. Informe general del estado de la ciencia y la tecnología. México: CONACYT, 2007.

CONACYT. Informe general del estado de la ciencia y la tecnología. México: CONACYT, 2010.

CONACYT. Informe general del estado de la ciencia y la tecnología. México: CONACYT, 2011.

CONACYT. Indicadores de actividades científicas y tecnológicas. México: CONACYT, 2009.

IMPI. Informe Anual 1994-1996. México: Instituto Mexicano de la Propiedad Industrial, 1996. Disponible en: http://www.impi.gob.mx/wb/IMPI/informe_anual_19941996.

IMPI. Informe Anual 2010. México: Instituto Mexicano de la Propiedad Industrial, 2010. Disponible en: http://www.impi.gob.mx/wb/IMPI/informe_anual_2010_v3.

IMPI. IMPI en cifras 2012. Cifras 1993 - Enero/Junio de 2012. México: Instituto Mexicano de la Propiedad Industrial, 2010. Disponible en: http://www.impi.gob.mx/wb/IMPI/ impi_en_cifras2. 
INEGI. Indicadores de actividades científicas y tecnológicas: Edición de bolsillo. México: INEGI, 2011. Disponible en: http://www.siicyt.gob.mx/siicyt/cms/paginas/Estadistica. jsp.

INEP. Censo da Educação Superior 2010 (Sinopses Educação Superior). Brasilia: Instituto Nacional de Estudos e Pesquisas Educacionais Anísio Teixeira, 2010. Disponible en: http://portal.inep.gov.br/superior-censosuperior-sinopse.

JIMÉNEZ Nájera, Yuri. Campo de la educación superior y regulación del trabajo académico en México: la participación de los académicos en la construcción social del orden político-laboral universitario (el caso de la UNAM: 1910-2007). 2010. Tesis (Doctorado en Ciencias Políticas y Sociales con orientación en Sociología) - Facultad de Ciencias Políticas y Sociales, Universidad Nacional Autónoma de México.

MORENO-BRID, Juan Carlos y RUIZ-NÁPOLES, Pablo. La educación superiory el desarrollo económico en América Latina. Santiago de Chile: CEPAL, 2009.

UNESCO. Clasificación Internacional Normalizada de la Educación (CINE) 1997. UNESCOUIS, 2006. Disponible en: http://www.uis.unesco.org/Library/Documents/isced97-es. pdf.

UNESCO. Compendio Mundial de la Educación 2011. Paris: UNESCO, 2011c.

UNESCO. Informe de Seguimiento de la Educación para Todos en el Mundo. Una crisis encubierta: conflictos armados y educación. Primera edición. París: UNESCO, 2011b.

UNESCO. L'UNESCO et l'éducation. «Toute personne a droit à l'éducation». Paris: UNESCO, 2011 a.

UNESCO. Rapport mondial sur l'educatión 1998. Paris: UNESCO, 1998.

VV. AA. Hacia una agenda nacional de Ciencia, Tecnología e Innovación. México: UNAM, 2012.

ARMANDO ALCÁNTARA SANTUARIO: é pesquisador da Universidad Nacional Autónoma de México. É Titular "A" com dedicação integral no Centro de Estudios sobre la Universidad. É Doutor em Educação com especialidade em Ciências Sociais e Educação Comparada pela Universidade da Califórnia (1999).

YURI JIMÉNEZ NÁJERA: é professor e pesquisador da Universidad Pedagógica Nacional (UPN). É Professor Titular com dedicação integral da Academia de Sociología de la Educación. 